\title{
WHAT WON'T ESCAPE SORITES ARGUMENTS
}

\section{By PAtrick Grim}

DETER UNGER and Samuel C. Wheeler have recently used sorites arguments - adaptations of Eubulides' argument of the heap in order to argue against the existence of swizzle sticks, stones, tables, people, and Peter Unger ([2], [3], [4], [5], [6]). Quine, too, takes sorites arguments of this sort seriously, and proposes that we abandon a wide range of apparently familiar objects in order to maintain a principle of bivalence ([1]).

Even those who have accepted these nihilistic conclusions, however, have thought that sorites arguments can be avoided fairly easily. Vague ordinary terms such as 'swizzle stick' and 'stone' fall victim to sorites arguments, and thus perhaps we must concede that there are no swizzle sticks or stones. But it has been assumed that we can easily introduce precise replacements for these terms which will escape sorites arguments. Unger, for example, anticipates a 'rather small' departure from common sense in the development of 'better, precise ideas' with which to replace the ordinary vague concepts which fall victim to sorites arguments ([4], p. 128). The only difficulty Unger discusses in this regard is that of deciding where to draw the line in a suitable replacement for, say, our ordinary term 'log';

With respect to atomic removals ... at a given juncture in a given case, there are millions of removals which seem quite innocuous and favorable. The item resulting from one such does not seem any more 'loggy' than that resulting from any other of them. Which steps are to be ruled out, and why? ([4], pp. 149-50).

Quine proposes precise replacement as follows:

When we do reach the point of positing numbers and plying their laws, then is the time to heed the contradictions and to work the requisite precision into the vague terms we learned by ostension. We arbitrarily stipulate, perhaps, how few grains a heap can contain and how compactly they must be placed. What had been observation terms are arbitrarily reconstructed, on pain of paradox, as theoretical terms whose application may depend in marginal cases on protracted tests and indirect inferences. The sorites paradox is one imperative reason for precision in science, among others ([1], p. 92).

In what follows I hope to show that sorites arguments, if taken seriously, are not to be avoided as easily as Quine and Unger suggest. The types of precise replacements which are usually envisaged would not escape sorites arguments. Any real escape, moreover, would demand a more complete and more drastic break from common talk of common things than those sympathetic to sorites arguments have generally anticipated. 
Consider a typical sorites argument, taken from Unger:

Here is an indirect argument to deny alleged swizzle sticks, those supposedly popular swizzle stirrers. We note that the existential supposition:

(1) There is at least one swizzle stick,

is inconsistent with the propositions we mean to express as follows:

(2) If anything is a swizzle stick, then it consists of more than one atom, but of only a finite number.

(3) If anything is a swizzle stick, then the net removal from it of one atom, or only a few, in a manner most innocuous and favorable, will not mean the difference as to whether or not there is a swizzle stick there.

Supposing (1) and (2), by (3) we get down below two atoms and still say that a swizzle stick is there. That contradicts (2). The only way to maintain (2) and (3), while being consistent, is to deny the existence of those sticks ([3], p. 519).

So much for swizzle sticks, at least if we take the argument seriously enough to maintain bivalence and to accept (2) and (3) on grounds of linguistic intuitions. So much, too, for stones, tables, and people, for it is clear that similar arguments could be constructed against these.

Here precise replacements offer a promise of escape, however. Following Quine's and Unger's suggestions regarding limited-grain heaps and atomically-specified logs, we might propose precise '1-billion-atom swizzle sticks' as suitable replacements for vague 'swizzle sticks' simpliciter. '1-billion-atom swizzle sticks' would seem, at least at first glance, to escape sorites arguments. For in order to construct an argument against the existence of 1-billionatom swizzle sticks analogous to Unger's argument above, it appears, we would need a premise (4):

(4) If anything is a 1-billion-atom swizzle stick, then the net removal from it of one atom, or only a few, in a manner most innocuous and favorable, will not mean the difference as to whether or not there is a 1-billion-atom swizzle stick there.

But (4), unlike its predecessor (3), is obviously false; removal of even a single atom will mean the difference as to whether or not we have a 1-billion-atom swizzle stick. Without that crucial premise, it appears, sorites arguments will not succeed against 1-billion-atom swizzle sticks.

Sorites arguments are not to be avoided so easily, however. Consider a slight variant on Unger's argument, designed to show the non-existence of 1-billion-atom swizzle sticks. We first introduce the existential supposition: 
(5) There is at least one 1-billion-atom swizzle stick,

and note that this is inconsistent with the following:

(6) If something is a 1-billion-atom swizzle stick, then something is a swizzle stick and is composed of 1 billion atoms.

(2) If anything is a swizzle stick, then it consists of more than one atom, but of only a finite number.

(3) If anything is a swizzle stick, then the net removal from it of one atom, or only a few, in a manner most innocuous and favorable, will not mean the difference as to whether or not there is a swizzle stick there.

Here (2) and (3) are the premises of Unger's original argument. (6) is an added premise which seems clearly true, since a 1-billion-atom swizzle stick would be a swizzle stick, composed of one billion atoms. But if we are to maintain consistency while accepting (6), (2), and (3), then we must deny (5), and with it the existence of 1-billion-atom swizzle sticks.

We might also argue for this conclusion somewhat less directly. Our original hope in introducing 1-billion-atom swizzle sticks as precise replacements was that (7) would be true:

(7) Sorites arguments fail to establish the non-existence of 1-billion-atom swizzle sticks.

But if this were true, together with the same apparently innocuous premise as before:

(6) If something is a 1-billion-atom swizzle stick, then something is a swizzle stick and is composed of 1 billion atoms,

we would have to conclude that:

(8) Sorites arguments fail to establish the non-existence of swizzle sticks.

If we follow Quine, Unger, and Wheeler in denying the existence of swizzle sticks on the basis of sorites arguments, then we will have to deny (8). Given the validity of the argument above, if we insist that (8) is false, we must deny (6) or (7). (6) is not a likely candidate. So we must deny (7) and concede that sorites arguments suceed in establishing the non-existence of 1-billion-atom swizzle sticks.

This conclusion should not be too surprising, despite the temptation to think, with Quine, that we can merely 'work the requisite precision into the vague terms that we learned by ostension' ([1], p. 92). For if there really are no heaps or swizzle sticks, there are no heaps of specific numbers of grains or swizzle sticks of precise numbers of atoms either. 
1-billion-atom swizzle sticks are vulnerable to sorites arguments because they are still swizzle sticks, and are still specified using a term, 'swizzle stick', which falls victim to sorites arguments. If precise replacement is to offer an escape from sorites arguments, then, we at least shall have to be a bit more subtle in our introduction of precision. Let us thus attempt more careful specification of a precise replacement designed to escape sorites arguments. Without actually constructing the required definitions, we might outline the following strategy for defining 'gizzle gick', intended as a replacement for 'swizzle stick'.

Were we attempting to introduce a precise replacement for 'cubical object', we might begin with a mathematical specification for a perfect cube. In the present case we might start with a similarly ideal 'mathematically perfect swizzloid'. Imagine, if you will, a paradigm swizzle stick. A suitable definition for 'mathematically perfect swizzloid' would be one which specifies the abstract shape of that imagined paradigm in purely mathematical terms, much as a mathematical specification of a perfect cube specifies the abstract shape of an imagined cube.

'Gizzle gicks' could now be defined in terms of a specific range of physical approximations to mathematically perfect swizzloids of, say, five inches in length. We might first define 'earthly perfect 5 " plastic swizzloids' as non-empty sets of plastic molecules at a specific time, temperature, pressure, etc., which approximate the requirements of a mathematically perfect swizzloid as closely as is physically possible. Here further specifications would undoubtedly be required, but let us assume that with patience these could be included in our definition. 'Gizzle gicks' might then be defined so as to allow a specific range of variation. Something is a gizzle gick, we might propose, if and only if it either is an earthly perfect $5^{\prime \prime}$ plastic swizzloid or is a non-empty set of plastic molecules at the specified time, temperature, pressure, etc., which would result in an earthly perfect $5^{\prime \prime}$ plastic swizzloid by the addition of one hundred molecules or less, properly placed.

By some such elaborate means, it appears, we might be able to introduce an appropriate replacement for 'swizzle stick' without using in our definitions any term against which sorites arguments will prove effective. Plastic molecules would not themselves seem liable to gradual decomposition in the manner of sorites arguments, and non-empty sets of such molecules have a clearly specified lower limit; one molecule or more is necessary and sufficient for a nonempty set. If we take sorites arguments seriously, we will have to do without swizzle sticks. But gizzle gicks will stir our drinks (or ginks) equally well.

But will even this more elaborate introduction of precision offer an escape from sorites arguments? Perhaps not. For consider the 
following argument against the existence of gizzle gicks. We start with the existential supposition that

(9) There is at least one gizzle gick,

and note that this is inconsistent with:

(10) If anything is a gizzle gick, that is sufficient for it to be a swizzle stick, in the ordinary sense of the term.

(11) If anything is a swizzle stick, in the ordinary sense of the term, then it consists of more than one atom, but of only a finite number.

(12) If anything is a swizzle stick, in the ordinary sense of the term, then the net removal from it of one atom, or only a few, in a manner most innocuous and favorable, will not mean the difference as to whether or not there is a swizzle stick there, in the ordinary sense of the term.

Here (11) and (12) differ from Unger's original (2) and (3) only in the addition of 'in the ordinary sense of the term', which was clearly intended anyway. (10) is a new premise, but seems at least as compelling of assent as (11) and (12). If we construct the definitions suggested above properly, we will have defined 'mathematically perfect swizzloid' on the pattern of an imagined paradigm swizzle stick, and will have defined 'gizzle gicks' in terms of sets of plastic molecules approximating closely the requirements of a mathematically perfect $5^{\prime \prime}$ swizzloid. So were I now to set a gizzle gick, suitably defined, before you, and were I to ask you whether that object would qualify as a swizzle stick in the ordinary sense of the term, I think you would have to answer 'yes'. Gizzle gicks have been specified in such a way that, were there such things, they would clearly qualify as swizzle sticks in the ordinary sense of the term. It is true that the plausibility of (10) relies on linguistic intuitions concerning 'swizzle stick'. But the same is true of (11) and (12), and of their predecessors (2) and (3). If we are to accept (2) and (3) in Unger's original argument on grounds of linguistic intuitions, there seems little reason not to extend (10) the same courtesy.

If we accept (10) through (12), however, we cannot consistently maintain (9) as well. Thus despite our care in constructing definitions with an eye to precise replacements, we must concede that there are no gizzle gicks.

We can also offer a more indirect argument for the same conclusion:

(13) Sorites arguments fail to establish the non-existence of gizzle gicks.

(10) If anything is a gizzle gick, that is sufficient for it to be a swizzle stick, in the ordinary sense of the term.

(14) Sorites arguments fail to establish the non-existence of swizzle sticks. 
With Wheeler, Unger, and Quine, we have assumed (14) to be false. But (13) and (10) offer a valid argument for (14), so we must deny either (10) or (13). (10), for reasons given, seems a poor choice for denial. So it appears that we must deny (13). Despite whatever care we might take in constructing definitions such as those suggested above, it appears that precise replacements of this sort will not escape sorites arguments.

\section{III}

What might we offer as precise replacements which would escape sorites arguments? Here prospects seem significantly slimmer than proponents of sorites arguments have suggested. In order to escape arguments such as those presented above, any precise replacement would have to avoid analogues of (10). Thus nothing could escape sorites arguments the existence of which were sufficient for the existence of any of the common objects which seem to fall victim to sorites arguments. Nothing offered by way of precise replacement could, for example, qualify as a large object, a small object, a red object, or a non-red object, for each of these is subject to sorites arguments.

If we are still to hope for precise replacements which will escape sorites arguments, they will not be precise replacements of the simple sort which Quine and Unger suggest. What would be required would be not the mere addition of precision to existing terms, nor the 'rather small' changes in common thought which Unger envisages, but rather a fairly drastic departure from our common talk of common objects.

State University of New York at Stony Brook C PATRICK GRIM 1982

\section{REFERENCES}

[1] W. V. Quine, 'What Price Bivalence?', Journal of Philosophy LXXVII (1981), pp. 90-5.

[2] Peter Unger, 'I Do Not Exist'. In Perception and Identity, ed. G. F. MacDonald, Ithaca, New York: Cornell University Press, 1979, pp. 235-51.

[3] 'Skepticism and Nihilism', Nous 14 (1980), pp. 517-45.

$\left[\begin{array}{l}4 \\ 5\end{array}\right]$ 'There Are No Ordinary Things', Synthese 41 (1979), pp. 117-54. (1979), pp. 177-222.

[6] Samuel C. Wheeler, 'On That Which Is Not', Synthese 41 (1979), pp. 155-73. 